\title{
ENTRE EL MACIZO DEL DESEADO Y LA MARGEN NORTE DE LA CUENCA DEL RÍO SANTA CRUZ (PATAGONIA, ARGENTINA): ANÁLISIS GEOQUÍMICO DE ARTEFACTOS DE OBSIDIANA Y MODELOS DE CIRCULACIÓN HUMANA
}

NORA V. FRANCO ${ }^{a}$, MICHAEL D. GLASCOCK ${ }^{b}$, BRANDI L. MACDONALD ${ }^{\mathrm{b}}$ \& BRENDA L. GILIO

\section{RESUMEN}

El objetivo de este trabajo es evaluar la posibilidad de la existencia de una fuente desconocida de obsidiana entre el sur del Macizo del Deseado y la margen norte de la cuenca del río Santa Cruz (Patagonia, Argentina). La alta frecuencia de obsidiana en algunos sectores de este espacio y la mención de pobladores locales acerca de la existencia de fuentes desconocidas hasta el momento llevaron a la necesidad de explorar esta hipótesis. Se realizaron análisis geoquímicos mediante fluorescencia de rayos X a una muestra de 20 artefactos de obsidiana. Los resultados indican una composición geoquímica similar a las fuentes secundarias de Pampa del Asador, su cono aluvial y 17 de Marzo o sectores intermedios entre ellas, lo que debilita la hipótesis acerca de la existencia de una fuente de obsidiana con una composición diferente en este sector del espacio. Por otra parte, a efectos de comenzar a evaluar cuál de las fuentes habría sido utilizada en cada caso, se hicieron análisis de frecuencia artefactual y se modelaron caminos óptimos entre cañadones que desembocan al norte del río Santa Cruz y las fuentes de obsidiana negra conocidas. Si bien se requieren análisis adicionales, los resultados sugieren la utilización de obsidiana de 17 de Marzo en los espacios más orientales (cañadón Yaten Guajen).

PALABRAS CLAVE: Patagonia, cazadores-recolectores, fuentes de obsidiana, análisis geoquímicos, caminos óptimos.

\section{BETWEEN THE DESEADO MASSIF AND THE NORTH SANTA CRUZ RIVER BASIN (PATAGONIA, ARGENTINA): GEOCHEMICAL ANALYSIS ON OBSIDIAN ARTIFACTS AND HUMAN CIRCULATION MODELS}

\section{ABSTRACT}

The purpose of this paper is to analyze the possibility of the existence of an unknown obsidian source between the south of the Deseado Massif and the northern shore of the Santa Cruz River

a IMHICIHU-CONICET / Universidad de Buenos Aires, FFyL, Saavedra 15, 5to Piso, Ciudad de Buenos Aires, C.P. 1083, Argentina. \nvfranco2008@gmail.com

b Archaeometry Laboratory, University of Missouri Research Reactor, Columbia, Missouri, 65211, Estados Unidos. glascockm@missouri.edu; macdonaldb@missouri.edu

c CIT Santa Cruz CONICET-UNPA, Lisandro de la Torre 860, Río Gallegos, Santa Cruz, C.P. 9400, Argentina. brendagilio@yahoo.com.ar 
basin (Patagonia, Argentina). The high frequency of obsidian in some of the analyzed spaces and the information provided by local inhabitants about the existence of an unknown obsidian source in the area, pointed to the need of evaluating this hypothesis. Geochemical analysis, through X-ray fluorescence, were performed on 20 obsidian artifacts recovered in different spaces of the study area. The results indicate a geochemical composition similar to the secondary sources of Pampa del Asador, its alluvial cone, and 17 de Marzo or intermediate sectors between them, which weakens the hypothesis of the existence of an unknown obsidian source with a different composition in this area. In addition, in order to evaluate which of the obsidian sources was used, obsidian artifacts frequencies were utilized and least cost paths were modeled between canyons in the northern shore of Santa Cruz river and the known black obsidian sources. Although further analysis need to be performed, results suggest the utilization of the 17 Marzo source in easterly spaces (Yaten Guajen canyon).

KEY WORDS: Patagonia, hunter-gatherers, obsidian sources, geochemical analysis, least cost paths.

\section{ANTECEDENTES GENERALES}

El sector localizado entre el sur del Macizo del Deseado y la cuenca del río Santa Cruz (Fig. 1), con ocupaciones humanas iniciales de ca. 10.800 y 7.700 años A.P. respectivamente (Franco et al. 2007 a, b, 2010; Franco, 2013; Franco et al. 2014; Brook et al. 2015; Franco et al. 2019), presenta frecuencias variables de artefactos confeccionados sobre obsidiana negra. Llamativamente, éstos son más abundantes en los cañadones basálticos localizados al norte del río Santa Cruz (a una distancia mínima de 240 km de Pampa del Asador), que en el Macizo del Deseado (a $120 \mathrm{~km}$ de ésta), un hecho que podría estar relacionado con la existencia de rocas silíceas de alta calidad en el último de estos espacios (Franco et al. 2015), además de poder vincularse con la forma de circulación humana en ellos. La distancia de $240 \mathrm{~km}$ es mayor que la esperada para rangos de acción en estas latitudes sobre la base de información etnográfica y etnoarqueológica (Binford, 1980; Kelly, 2013; entre otros). Si bien podría pensarse que esta materia prima podría provenir también de la fuente recientemente localizada de 17 de Marzo (Franco et al. 2017), en este caso los nódulos son más pequeños y la distancia euclidiana a ésta es de aproximadamente $166 \mathrm{~km}$, apenas por debajo de las distancias de $175 \mathrm{~km}$ estimadas para movimientos logísticos de grupos cazadores-recolectores en estas latitudes (Kelly, 2011).

La importancia de la fuente secundaria de obsidiana negra de Pampa del Asador -PDA- (Fig. 1), ubicada al noroeste de la provincia de Santa Cruz, ha sido reconocida desde hace tiempo (Espinosa \& Goñi, 1999; Stern, 2000, 2004; Franco, 2004; entre otros). Presenta una extensión sudoeste-noreste de $80 \mathrm{~km}$ por un ancho medio de $15 \mathrm{~km}$ y altitudes entre 650 y 1.100 msnm e incluye el cerro Pampa, cerro Bayo, sitio Musters y distintas mesetas basálticas (Meseta Pobre, Meseta Olnie, Meseta La Cantera, etc.) (Stern et al. 1995, a y b; Espinosa \& Goñi, 1999; Stern, 2000, 2004; Belardi et al. 2006). Los nódulos presentan tamaños máximos de $100 \mathrm{~mm}$, aunque la mayoría tiene dimensiones inferiores a $60 \mathrm{~mm}$ (Cassiodoro et al. 2015). Los nódulos son de color negro, aunque Stern (1999) menciona que tienen también bandas más claras grises y franjas de oxidación rojas. Durante mucho tiempo se consideró como la única fuente de obsidiana negra en Patagonia Centro-Sur (Espinosa \& Goñi, 1999; Stern, 2000, 2004; Franco, 2004; Ambrústolo et al. 2012; Cueto et al. 2016), aunque más recientemente se han descubierto otras fuentes. Se trata, en primer lugar, de su abanico aluvial (CAPDA), ubicado hacia el este, con cotas entre 400 y 740 m. Los tamaños máximos de los nódulos son inferiores a $58 \mathrm{~mm}$ (Belardi et al. 2006). Por otra parte, el descubrimiento de la fuente distal de 17 de Marzo (Fig. 1), a una distancia de más de 100 km al este, sugiere que pueden existir, además de ésta, otras fuentes secundarias entre estos espacios. En el caso de 17 de Marzo, los tamaños de los nódulos son inferiores a $48 \mathrm{~mm}$ (Franco et al. 2017). Por 


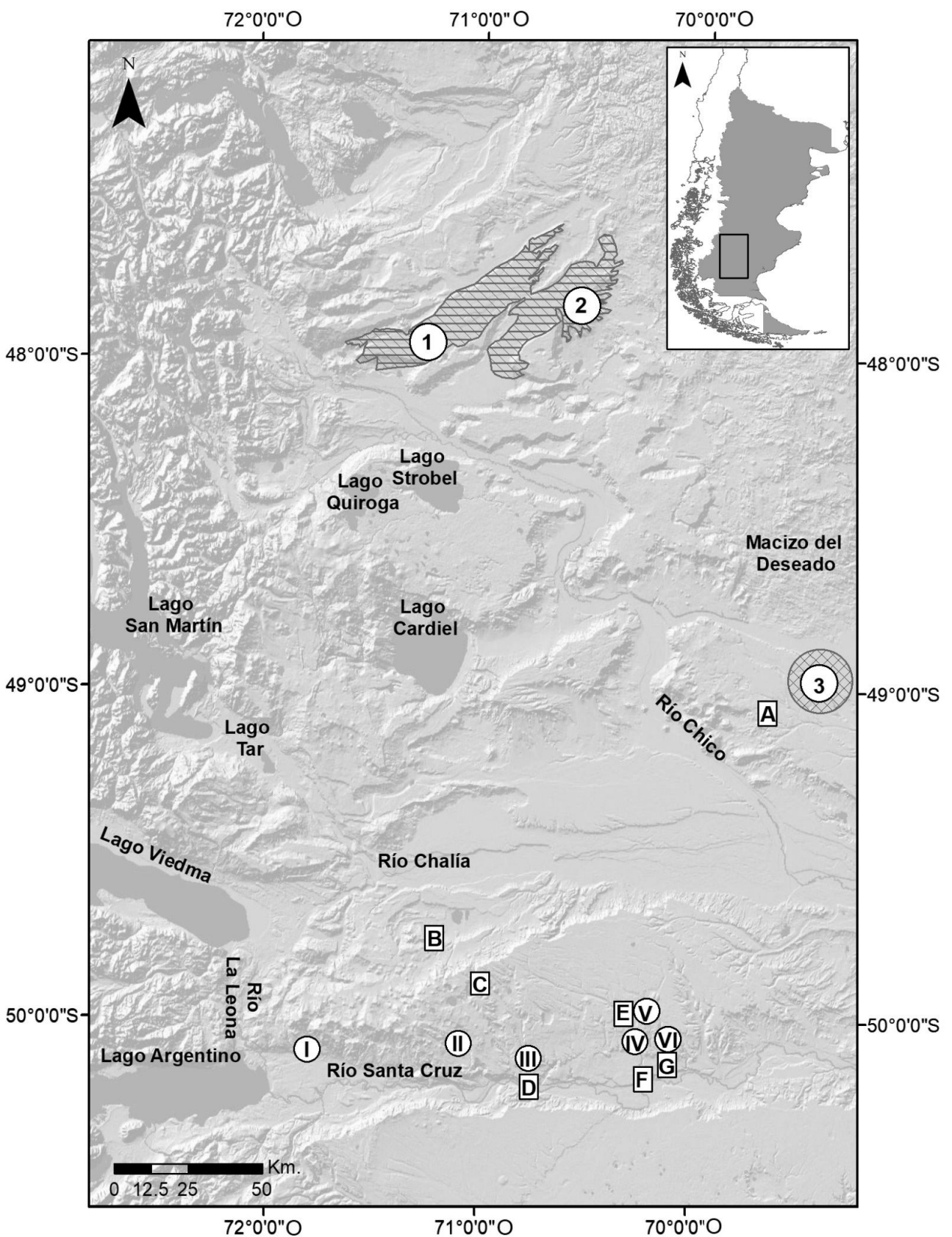

Fig. 1. Ubicación del área de estudio, fuentes de obsidiana negra conocidas (en números arábigos), cañadones involucrados en el análisis (en números romanos) y muestras arqueológicas de obsidiana negra analizadas geoquímicamente (en letras). Referencias: 1: PDA, 2: CAPDA, 3: 17 de Marzo; I: La Laurita-

El Rincón, II: El Lechuza, III: El Cordero, IV: Yaten Guajen, V: Mercerá, VI: Kolian Kaike; A: Cardiel Chico, B: María Elena, C: La Betty, D: La Fructuosa, E: Yaten Guajen, F: La Barrancosa, G: Güent Aike.

el momento, contrario a Nami y colaboradores (2018), tendemos a descartar la existencia de una fuente de obsidiana a lo largo de la costa, ya que las muestras analizadas se obtuvieron en sitios arqueológicos (Punta Medanosa) y sólo se ofreció información muy general acerca de la procedencia del resto de los ejemplares analizados (ver Franco et al. 2019). 
La circulación humana entre los espacios del oeste -incluidos PDA y el espacio próximo de la meseta del Strobel (Fig. 1)- y los cañadones basálticos al norte del río Santa Cruz (Fig. 1) ha sido postulada por diferentes autores sobre la base de similitudes en motivos grabados de arte rupestre (Gradin et al. 1979; Gradin, 2000, 2001; Belardi \& Goñi, 2006; Franco, 2008; Goñi et al. 2006, 2009; Re, 2010; Franco et al. 2014; entre otros). Gradin (1976) sugirió que estas semejanzas están relacionadas con la movilidad estacional, con mesetas que se utilizarian en verano y áreas ubicadas cerca de ríos o lagos, que se usarian en invierno. Por otra parte, Belardi y Goñi (2006) indican que estas semejanzas se relacionarían con la convergencia estacional, no necesariamente sincrónica, de poblaciones en el área de la meseta del Strobel. Por otro lado, también se ha sugerido la circulación entre el sur del Macizo del Deseado y los cañadones al norte del río Santa Cruz sobre la base de la presencia y características de rocas silíceas (Franco \& Cirigliano, 2009).

La presencia de obsidiana $\mathrm{u}$ otras materias primas en el registro arqueológico no implica necesariamente una adquisición directa, ya que su existencia también puede ser el resultado de la movilidad y/o interacción humana (entre otros, Renfrew, 1977; Ericson, 1984; Meltzer, 1989; Ingbar, 1994; Kelly, 2013; Pallo \& Borrero, 2015; Franco et al. 2015, 2017). Sobre la base de datos propios y los obtenidos por distintos investigadores utilizando muestras de superficie y estratigrafía, Pallo y Borrero (2015) indican que la frecuencia de obsidiana negra es alta (> 60\%) en sitios inmediatamente próximos y hasta $50 \mathrm{~km}$ de PDA, decreciendo en una curva fall-off a distancias de alrededor de 140 $\mathrm{km}$ o, a $125 \mathrm{~km}$ al punto más cercano a PDA, con porcentajes que oscilan entre el 60 y el 10\%. Más allá de esta distancia, no superan el 15\%. Sin embargo, mencionan que algunas áreas, como el lago San Martín, presentan frecuencias de obsidiana más altas que lo esperado (35\% a 48\%), lo que es atípico, como ya lo señalaron también Molinari y Espinosa (1999). A mayores distancias, el porcentaje de obsidiana decrece ( $<15 \%)$, alcanzando un nuevo umbral en los $400 \mathrm{~km}$, distancia a partir de la cual su presencia es mínima $(<1 \%)$.

Cabe señalar, por otra parte, que habitantes locales han sugerido la existencia de fuentes adicionales de obsidiana tanto en el Macizo del
Deseado (Aguerre, 2003) como en los cañadones al norte del río Santa Cruz (Franco, 2008) (Fig. 1). Por otro lado, Molinari y Espinosa (1999), sobre la base de las altas frecuencias de artefactos de obsidiana, sugieren la existencia de una fuente de esta materia prima aún no identificada, próxima al lago San Martín (Fig. 1).

En nuestro caso, era importante entonces evaluar si la alta frecuencia de obsidiana en los cañadones del norte del río Santa Cruz en comparación con aquélla presente en el Macizo del Deseado y en espacios al norte del río Chico, podía relacionarse con la circulación de poblaciones humanas o podía deberse a la existencia de una fuente de aprovisionamiento aún no descubierta. En este sentido, cabe señalar que los análisis geoquímicos practicados por Stern sobre tres muestras procedentes de este sector del espacio (Franco, 2008) señalaron también su semejanza geoquímica con las fuentes secundarias de PDA, su cono aluvial y 17 de Marzo. Era necesario ampliar la muestra para analizar si éste continuaba siendo el caso. Por otra parte, era importante evaluar también, en el caso de que proviniera de éstas, cuál era la fuente de obtención más probable.

\section{MUESTRA Y METODOLOGÍA}

Se analizó un total de 20 muestras arqueológicas (Tabla 1), seleccionando ejemplares de distinta procedencia $y$, en la medida de lo posible, artefactos no retocados, con corteza, cuyas dimensiones se acercaran a las mayores en la zona y que presentaran variaciones en color, que pudieran estar incluidas dentro de las disponibles en las fuentes mencionadas. La selección de los artefactos de tamaño más grande se basó en la expectativa de que, a mayor cercanía de la fuente, la dimensión fuera mayor, de acuerdo con modelos fall-off de aprovisionamiento de materia prima.

Los sectores de procedencia de las muestras analizadas fueron indicados con una letra mayúscula para representar su localización espacial en la tabla 1 y en las figuras. Su mayoría (15 muestras correspondientes a las referencias espaciales D, E, F y G en la Tabla 1 y Fig. 1) proviene de los cañadones localizados al norte del río Santa Cruz, mientras que cuatro proceden del espacio abierto entre ellos y el río Chalía 
Tabla 1. Características de las muestras de artefactos arqueológicos analizados geoquímicamente. Referencias: Indet: indeterminada.

\begin{tabular}{|c|c|c|c|c|c|c|c|c|}
\hline $\begin{array}{c}\text { Sector de } \\
\text { procedencia } \\
\text { de la muestra }\end{array}$ & $\begin{array}{l}\text { Referencia } \\
\text { espacial }\end{array}$ & $\begin{array}{c}\text { Cantidad de } \\
\text { muestras }\end{array}$ & $\begin{array}{c}\text { Longitud } \\
\text { máxima } \\
(\mathrm{mm})\end{array}$ & $\begin{array}{l}\text { Porcentaje de } \\
\text { corteza }\end{array}$ & $\begin{array}{l}\text { Tipo de } \\
\text { muestra }\end{array}$ & Forma base & Entero & Color \\
\hline Cardiel Chico & A & 1 & 28 & $50 \%$ & Núcleo & Guijarro & Sí & Negro \\
\hline María Elena & B & 1 & 30 & $50 \%$ & Cuchillo & Lasca & No & Negro \\
\hline La Betty & $\mathrm{C}$ & 3 & $29-39$ & $\begin{array}{c}50 \% \text { ( } 1 \text { caso) } \\
0 \% \text { (2 casos) }\end{array}$ & $\begin{array}{c}\text { Raedera (1), } \\
\text { lasca (1) y } \\
\text { núcleo (1) }\end{array}$ & $\begin{array}{l}\text { Núcleo, } \\
\text { raspador, } \\
\text { lasca }\end{array}$ & $\begin{array}{l}\text { Sî (2), No } \\
\text { (1) }\end{array}$ & Negro \\
\hline La Fructuosa & $\mathrm{D}$ & 3 & $24-27$ & $\begin{array}{c}25 \%(2 \\
\text { casos), } \\
0 \% \text { (1 caso) }\end{array}$ & Lascas (3) & Indet. (3) & $\begin{array}{l}\text { Sî (1), } \\
\text { No (2) }\end{array}$ & $\begin{array}{c}\text { Negro } \\
\text { (1), Gris } \\
\text { (2) }\end{array}$ \\
\hline $\begin{array}{l}\text { Yaten } \\
\text { Guajen }\end{array}$ & E & 4 & $20-32$ & $\begin{array}{c}75 \%(1) \\
50 \%(1) \\
0 \%(2)\end{array}$ & $\begin{array}{c}\text { Raedera (1), } \\
\text { lascas (3) }\end{array}$ & Indet. (4) & $\begin{array}{l}\text { Sí (3), } \\
\text { No (1) }\end{array}$ & $\begin{array}{l}\text { Negro } \\
(4)\end{array}$ \\
\hline La Barrancosa & $\mathrm{F}$ & 7 & $28-44$ & $\begin{array}{c}0 \% \text { ( } 1 \text { caso), } \\
25 \%(3 \\
\text { casos), } 75 \% \\
(2 \text { casos), } \\
100 \% \text { (1 } \\
\text { caso) }\end{array}$ & $\begin{array}{l}\text { Lascas (6), } \\
\text { artefacto no } \\
\text { determinado } \\
\text { (1) }\end{array}$ & $\begin{array}{c}\text { Guijarro (3), } \\
\text { indet. (4) }\end{array}$ & $\begin{array}{l}\text { Sí (6), } \\
\text { No (1) }\end{array}$ & $\begin{array}{l}\text { Negro } \\
(7)\end{array}$ \\
\hline Güent Aike & G & 1 & 32 & $0 \%$ & Lasca & Indet. & Sí & Gris \\
\hline
\end{tabular}

(María Elena -sector B- y La Betty -sector C-), y un último ejemplar corresponde al área basáltica ubicada al norte del río Chico -sector A- (Tabla 1, Fig. 1). Los tamaños de las muestras analizadas varían entre 20 y $44 \mathrm{~mm}$. Su mayor parte $-70 \%$ del total- corresponde a lascas (en su mayoría angulares pero también primarias, secundarias y de reducción bifacial), estando en general enteras $-70 \%$ del total-. Su mayoría $-65 \%$ del totalpresenta corteza.

Losartefactosseanalizaron por fluorescencia de rayos $\mathrm{X}(\mathrm{XRF})$ utilizando un espectrómetro XRF dispersivo de energía Bruker Tracer-5i en el MU Research Reactor. El instrumento funcionó a $50 \mathrm{kV}$ con una corriente de $35 \mu \mathrm{A}$ y cada muestra se analizó durante 60 segundos. Los elementos medidos incluyen potasio $(\mathrm{K})$, calcio $(\mathrm{Ca})$, titanio $(\mathrm{Ti})$, hierro $(\mathrm{Fe})$, cobre $(\mathrm{Cu})$, zinc $(\mathrm{Zn})$, galio $(\mathrm{Ga})$, $\operatorname{arsénico~}(\mathrm{As})$, rubidio $(\mathrm{Rb})$, estroncio $(\mathrm{Sr})$, itrio $(\mathrm{Y})$, circonio $(\mathrm{Zr})$, niobio $(\mathrm{Nb})$, bario $(\mathrm{Ba})$ y torio $(\mathrm{Th})$. El instrumento se calibró utilizando un conjunto de 40 muestras de fuentes de obsidiana que se caracterizaron previamente por análisis de activación de neutrones (NAA), espectrometría de masas de plasma acoplada inductivamente
(ICP-MS) y XRF. La colección de fuentes tiene una amplia gama de concentraciones (de menor a mayor) de elementos medibles por XRF (Glascock $\&$ Ferguson, 2012). Los datos resultantes se compararon con una base de datos sólida de fuentes de obsidiana conocidas de noroeste de Patagonia.

A los efectos de evaluar cuál era la fuente de aprovisionamiento que involucraba un menor costo de obtención, en primer lugar se modelaron los caminos óptimos de invierno y verano desde los lugares de recolección de las muestras analizadas geoquímicamente hacia las fuentes de obsidiana negra conocidas. Posteriormente, a los efectos de lograr un panorama más completo, se realizó un análisis incluyendo además información de cañadones cercanos para los que se posee datos de frecuencia de obsidiana negra cuantificada. Los cañadones implicados en este análisis fueron, de oeste a este (Fig. 1): La Laurita-El Rincón, El Lechuza, El Cordero (con su muestra denominada La Fructuosa), Yaten Guajen en su curso superior y medio (muestras denominadas Yaten Guajen) $y$ en su curso inferior (muestra La Barrancosa), Mercerá, además de Kolian Kaike (muestra 
Güent Aike). Los datos relativos a las frecuencias artefactuales (obtenidos en las publicaciones, en análisis propios y otros cedidos por Vetrisano) se compararon con las distancias euclidianas y aquéllas obtenidas mediante caminos óptimos o de menor costo energético entre los lugares de los que proviene la información y las fuentes de aprovisionamiento de obsidiana negra postuladas (PDA, CAPDA y 17 de Marzo). Los caminos óptimos hacen referencia a sectores del terreno que requieren menor esfuerzo en términos de energía invertida (costo) al ser recorridos por un individuo (Fairén Jiménez, 2004; Conolly \& Lake, 2006; Magnin, 2013; Pallo, 2016). La ruta de menor costo energético une dos o más puntos determinados con anterioridad (uno de origen y otro de destino) en función del costo mínimo de recorrido de una capa de fricción previamente confeccionada, donde la suma de valores de todas las celdas atravesadas sea la más baja y, por lo tanto, la que equivaldrá a un menor esfuerzo en el desplazamiento (López Romero, 2005). Dicha capa de fricción representa el costo de accesibilidad del terreno, es decir, la suma de los costos de fricción asignados a cada categoría de las variables ambientales que representan los valores de costo de una variable particular. En esta investigación se seleccionaron las siguientes variables: pendiente del terreno, mesetas basálticas, fuentes de agua (lagunas, lagos, arroyos, manantiales, etc.). En el modelo de circulación humana de invierno se sumó además una capa de carga nívea. Los caminos óptimos estacionales se modelaron a partir de diferentes procesos de análisis espacial mediante el software ArcMap 10. La pendiente del terreno y la capa de carga nívea se generaron a partir de un Modelo Digital de Elevación (MDE) con una grilla de 1 arco/segundo, aproximadamente de $30 \mathrm{~m}$ de resolución, desarrollado por el Instituto Geográfico Nacional de la República Argentina (IGN). Las mesetas basálticas se vectorizaron a partir de hojas geológicas de escala 1:250.000 desarrolladas por el Servicio Geológico Minero Argentino (SEGEMAR) (Gilio et al. 2016; Gilio, 2017). Las fuentes de agua se desarrollaron mediante capas vectoriales de cursos y cuerpos de agua provenientes del SIG250 del IGN de escala 1:250.000 (Base de Datos Geográfica, Instituto Geográfico Nacional, 2019).

\section{RESULTADOS}

\section{Características de la}

muestra y análisis geoquímicos

Entre las muestras analizadas se ha identificado la presencia de guijarros como formas base de artefactos ( 4 casos de un total de 8 formas base identificables, ver Tabla 1). Al menos uno de ellos (un núcleo con una sola extracción recuperado en Cardiel Chico, en los cañadones basálticos al norte del río Chico), debido a su pequeño tamaño, probablemente fue transportado como un guijarro a nuestra área de estudio. La presencia de pátina diferencial en uno de los casos sugiere la existencia de reclamación de artefactos previamente abandonados. En el caso de los cañadones al norte del río Santa Cruz, esto probablemente se deba a la falta de materia prima de calidad similar a la obsidiana para la talla (Franco et al. 2015).

Los resultados se presentan en un diagrama de dispersión de $\mathrm{Sr} / \mathrm{Rb}$ versus $\mathrm{Rb} /$ $\mathrm{Zr}$, que muestra la distribución de los artefactos de obsidiana y las fuentes y sub-fuentes conocidas (Fig. 2). Las muestras de artefactos proyectadas contra las tres fuentes de obsidiana de PDA producen asignaciones de fuentes satisfactorias para 19 de los 20 artefactos. El artefacto que no pudo ser asignado (NFSC04) se ubica en el gráfico cerca de Pampa del Asador-3 (Fig. 2) aunque no se pudo asignar con confianza a ninguna fuente conocida en la base de datos. Los resultados de los análisis efectuados indican entonces que todas las muestras provienen de una de las fuentes secundarias ya conocidas o de una de las fuentes probablemente ubicadas entre ellas. Los resultados obtenidos no sustentan la existencia de una fuente primaria o secundaria con una composición química diferente en los cañadones del norte del río Santa Cruz, a pesar de la alta frecuencia de obsidiana en este sector del espacio. Si hubiera otra fuente, tendría que tener la misma composición que PDA. De hecho, como PDA es una fuente secundaria, los guijarros disponibles allí pueden provenir de más de una fuente primaria.

El tamaño de los artefactos analizados, que es inferior a $44 \mathrm{~mm}$ (Tabla 1), no nos permite discriminar entre ninguna de las fuentes secundarias conocidas, ya que en todos los casos 


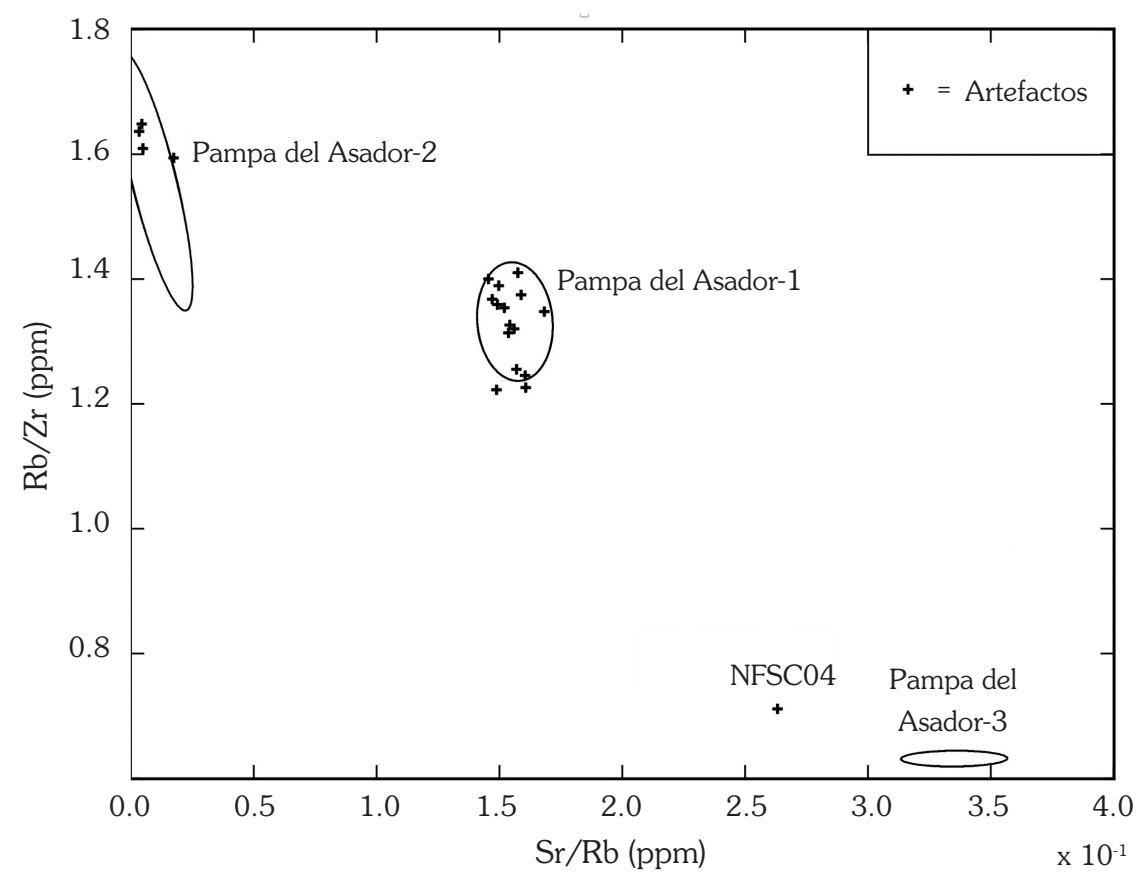

Fig. 2. Gráfico de dispersión de $\mathrm{Sr} / \mathrm{Rb}$ versus $\mathrm{Rb} / \mathrm{Zr}$ para artefactos de obsidiana procedentes del norte del río Santa Cruz, proyectados contra elipses de las subfuentes de Pampa del Asador con un nivel de confianza del 90\%.

los nódulos presentan mayores dimensiones. La alta frecuencia de utilización de obsidiana en los cañadones de la margen norte del río Santa Cruz está probablemente relacionada no sólo con los movimientos estacionales entre estos cañadones y PDA o mesetas cercanas (Gradin, 1976, 1983, 2001; Gradin et al. 1979; Belardi \& Goñi, 2006; Goñi et al. 2006, 2009), sino también con la disponibilidad de esta materia prima en la zona de 17 de Marzo y, potencialmente, en los espacios abiertos ubicados entre ésta y PDA (Fig. 1).

\section{Modelado de caminos \\ óptimos y frecuencias artefactuales}

Como se ha mencionado, a los efectos de evaluar cuál era la fuente de aprovisionamiento que involucraba menor costo de obtención, se modelaron primero los caminos óptimos entre los lugares de recolección de las muestras analizadas geoquímicamente y las fuentes de obsidiana negra conocidas, para luego realizar un análisis que incluyera además los cañadones cercanos $e$ información sobre la frecuencia artefactual.
En la Tabla 2 se indican los resultados del modelado de caminos y las distancias euclidianas entre las muestras analizadas $y$ las fuentes potenciales de aprovisionamiento conocidas. Éstas aumentan si, en lugar de considerarse las distancias euclidianas, se modelan las rutas óptimas, tanto en el caso de invierno como de verano (Tablas 2 y 3).

En todos los casos, los caminos óptimos son más largos en invierno que en verano. Esto se relaciona con que, en el caso del invierno, éstos no se dirigen directamente hacia las fuentes, sino que siguen por sectores bajos con ausencia o mínima presencia de carga nivea (Tabla 2, Figs. 3, 4 y 5). Cabe señalar que, mientras los caminos óptimos de verano poseen valores aproximadamente semejantes a los que involucra la distancia euclidiana, en invierno los caminos óptimos se prolongan 100 o más kilómetros para los casos de PDA y CAPDA, siendo la diferencia menor en algunos casos para la zona de 17 de Marzo.

Las distancias involucradas en los caminos óptimos son mayores que las esperadas la base de los tamaños de los sobre rangos de acción para grupos humanos localizados a estas latitudes, tanto 
Tabla 2. Distancia euclidiana y longitud de caminos óptimos entre los sectores de procedencia de las muestras de artefactos de obsidiana negra analizados geoquímicamente y las fuentes de obsidiana negra. Referencias: km: kilómetros; PDA: Pampa del Asador; CAPDA: Cono aluvial de Pampa del Asador; Refer.: referencia.

\begin{tabular}{lccccccccccc}
\hline $\begin{array}{c}\text { Sector de } \\
\begin{array}{c}\text { procedencia de la } \\
\text { muestra }\end{array}\end{array}$ & $\begin{array}{c}\text { Refer. } \\
\text { espacial }\end{array}$ & \multicolumn{4}{c}{ Distancia euclidiana (km) } & \multicolumn{3}{c}{$\begin{array}{c}\text { Caminos óptimos de } \\
\text { invierno (km) }\end{array}$} & \multicolumn{3}{c}{$\begin{array}{c}\text { Caminos óptimos de } \\
\text { verano (km) }\end{array}$} \\
\hline & & PDA & CAPDA & $\begin{array}{c}17 \text { de } \\
\text { Marzo }\end{array}$ & PDA & CAPDA & $\begin{array}{c}17 \text { de } \\
\text { Marzo }\end{array}$ & PDA & CAPDA & $\begin{array}{c}17 \text { de } \\
\text { Marzo }\end{array}$ \\
Cardiel Chico & A & 166 & 147 & 27 & 262 & 277 & 34 & 171 & 164 & 29 \\
María Elena & B & 197 & 216 & 154 & 439 & 455 & 250 & 209 & 226 & 164 \\
La Betty & C & 213 & 232 & 154 & 430 & 446 & 241 & 225 & 241 & 158 \\
La Fructuosa & D & 250 & 261 & 167 & 465 & 480 & 276 & 264 & 267 & 174 \\
Yaten Guajen & E & 233 & 238 & 127 & 385 & 400 & 196 & 249 & 252 & 134 \\
La Barrancosa & F & 258 & 262 & 146 & 414 & 429 & 225 & 280 & 273 & 153 \\
Güent Aike & G & 252 & 254 & 134 & 408 & 423 & 219 & 275 & 268 & 145 \\
\hline
\end{tabular}

para el caso de PDA como para su cono aluvial, con la única excepción de la muestra de Cardiel Chico. En cambio, las distancias en verano a 17 de Marzo se encuentran en todos los casos dentro del rango máximo esperado para cazadoresrecolectores en estas latitudes. En el caso de las muestras recuperadas en los espacios más orientales (La Fructuosa, La Barrancosa, Yaten Guajen y Güent Aike), los caminos óptimos de invierno en algunos casi duplican las distancias euclidianas involucradas, mientras que en verano el aumento en la distancia es menor. A manera de ejemplo, se grafican algunos casos aquí. En el caso de La Fructuosa, mientras en verano todos los caminos óptimos se dirigen hacia el norte, en invierno éstos siguen parcialmente el curso del río Santa Cruz hacia el este (Fig. 3), incrementando de esta manera la distancia recorrida.

Tanto en el caso del curso medio y superior del cañadón Yaten Guajen (Fig. 4) como en su curso inferior (La Barrancosa, Fig. 5), los caminos de verano también se dirigen directamente hacia el norte, mientras que en invierno siguen parcialmente el curso del río Chalía y Chico (en el caso de Yaten Guajen) y Santa Cruz (en el caso de La Barrancosa) (Figs. 4 y 5).

En la Tabla 3 se presentan las frecuencias de obsidiana negra provenientes de superficie y estratigrafía en distintos cañadones, obtenidas tanto en la bibliografía como en análisis de laboratorio. Éstas se relacionan con las distancias euclidianas y con aquéllas provenientes de caminos óptimos de invierno y verano hacia PDA, CAPDA y 17 de Marzo.

La información de superficie procedente de los cursos superior y medio de los cañadones muestra un decrecimiento en la frecuencia de obsidiana negra de oeste a este (Tabla 3). Ésta oscila entre un 13,17\% en el caso de La Laurita-El Rincón, un $12 \%$ en el caso del cañadón El Lechuza y un $9,6 \%$ en Yaten Guajen. Esto sugiere una mayor facilidad de acceso a la fuente en el caso de los espacios más occidentales, aunque debe tenerse en cuenta que, en el último caso, pobladores locales han hecho gran cantidad de recolecciones de superficie orientadas hacia la obsidiana negra, lo que provocaría un sesgo en su frecuencia (Franco et al. 2007a).

En el caso de La Laurita-El Rincón, la variación en distancia en verano oscila entre 222 y $278 \mathrm{~km}$, siendo los extremos 17 de Marzo y el CAPDA (Tabla 3). En invierno, la diferencia es mayor, oscilando entre 316 y $480 \mathrm{~km}$. Tanto los caminos de verano como de invierno hacia PDA pasan próximos a lagos de diferentes dimensiones (Fig. 6), los que podrían haber sido atractivos para los grupos humanos por la variedad de recursos que concentran (Belardi \& Goñi, 2006; Goñi et al. 2006; entre otros). Este es el caso de los lagos San Martín (a aproximadamente $18 \mathrm{~km}$ del camino óptimo), Tar (costa este) y Quiroga (10 km). Por otra parte, el camino de verano desde el cañadón 


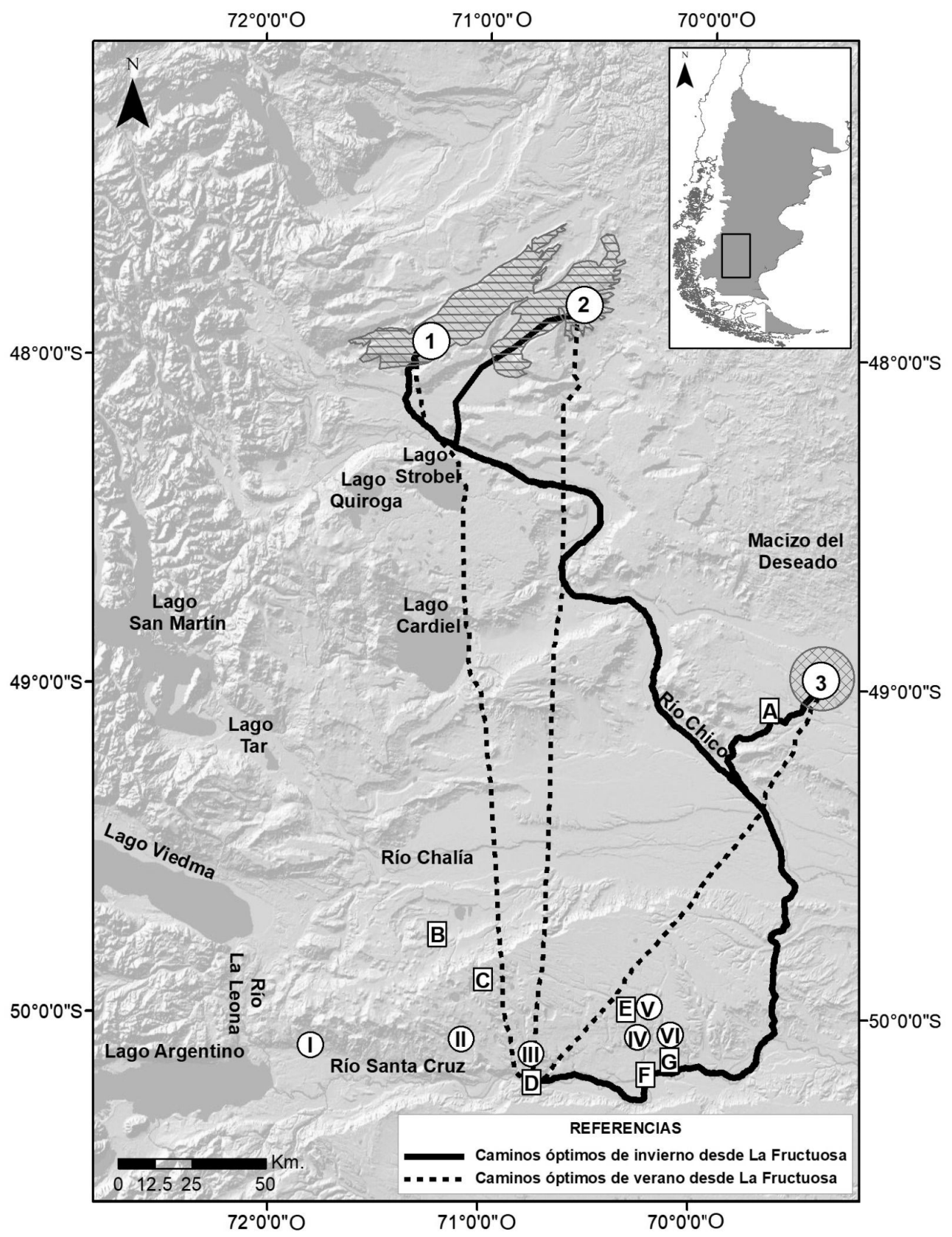

Fig. 3. Caminos óptimos de invierno y verano desde La Fructuosa. Referencias: 1: PDA, 2: CAPDA, 3: 17 de Marzo; I: La Laurita-El Rincón, II: El Lechuza, III: El Cordero; IV: Yaten Guajen, V: Mercerá, VI: Kolian Kaike; A: Cardiel Chico, B: María Elena, C: La Betty, D: La Fructuosa, E: Yaten Guajen, F: La Barrancosa, G: Güent Aike.

La Laurita-El Rincón hacia CAPDA, pasa por la costa este del lago Cardiel y coincide con el actual trazado de la ruta nacional 40 propuesta por Belardi y colaboradores (2009) como un corredor que habría conectado Pampa del Asador con los ríos Chico y Chalía -que serían la entrada a las cuencas de los lagos Tar y San Martín- y la margen noreste del lago Viedma. 


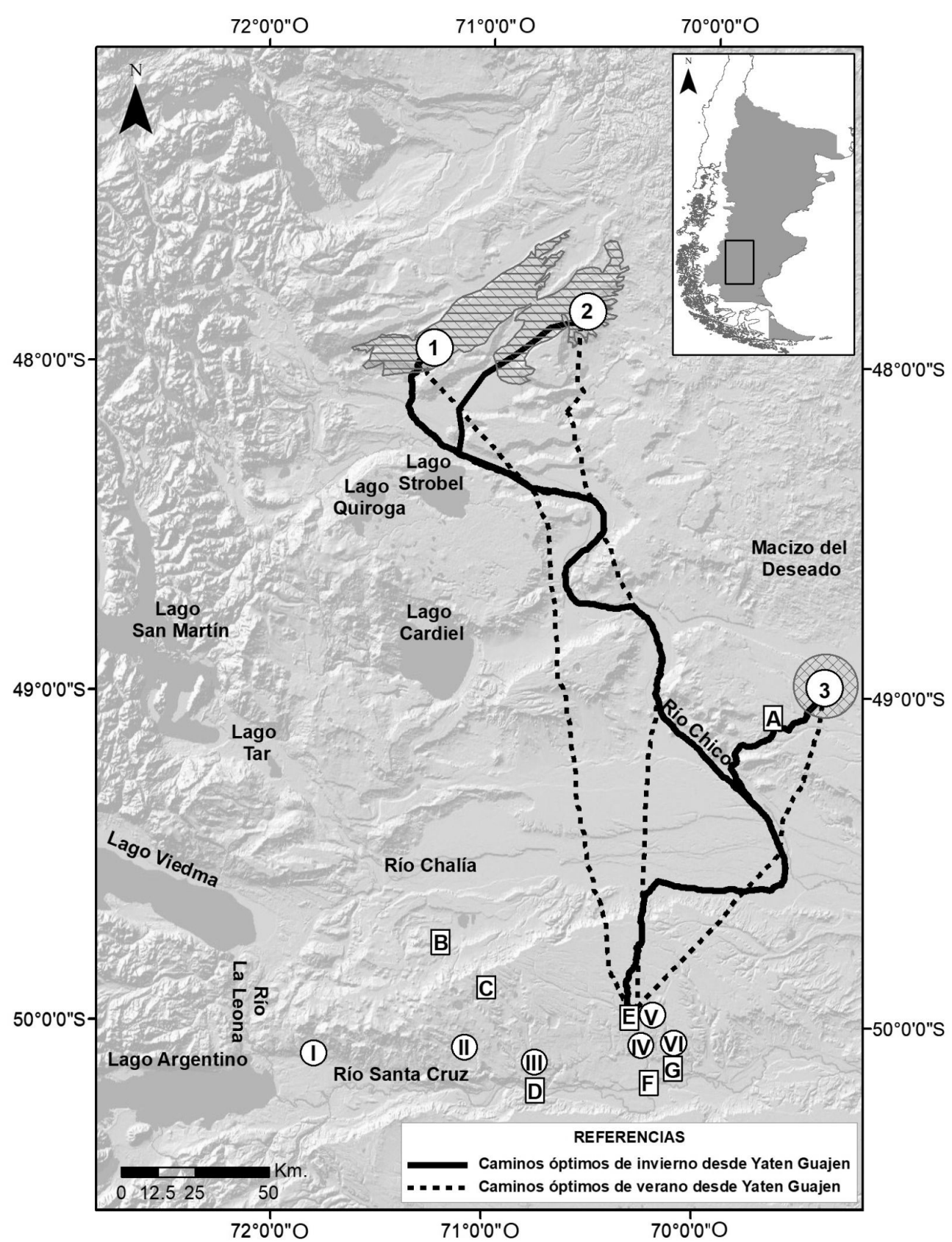

Fig. 4. Caminos óptimos de invierno y verano desde Yaten Guajen, curso superior y medio. Referencias: 1: PDA, 2: CAPDA, 3: 17 de Marzo; I: La Laurita-El Rincón, II: El Lechuza, III: El Cordero; IV: Yaten Guajen, V: Mercerá, VI: Kolian Kaike; A: Cardiel Chico, B: María Elena, C: La Betty, D: La Fructuosa, E: Yaten Guajen, F: La Barrancosa, G: Güent Aike.

En el caso del curso superior y medio de El Lechuza (Fig. 7), los caminos de verano oscilan entre 169 y $256 \mathrm{~km}$, siendo también los extremos 17 de Marzo y CAPDA. En invierno la variación es importante, siendo de $251 \mathrm{~km}$ hacia 17 de Marzo, $415 \mathrm{~km}$ el CAPDA y $441 \mathrm{~km}$ en el caso de PDA. En verano, el camino óptimo desde este sector a PDA pasa por la margen este de los lagos Cardiel 


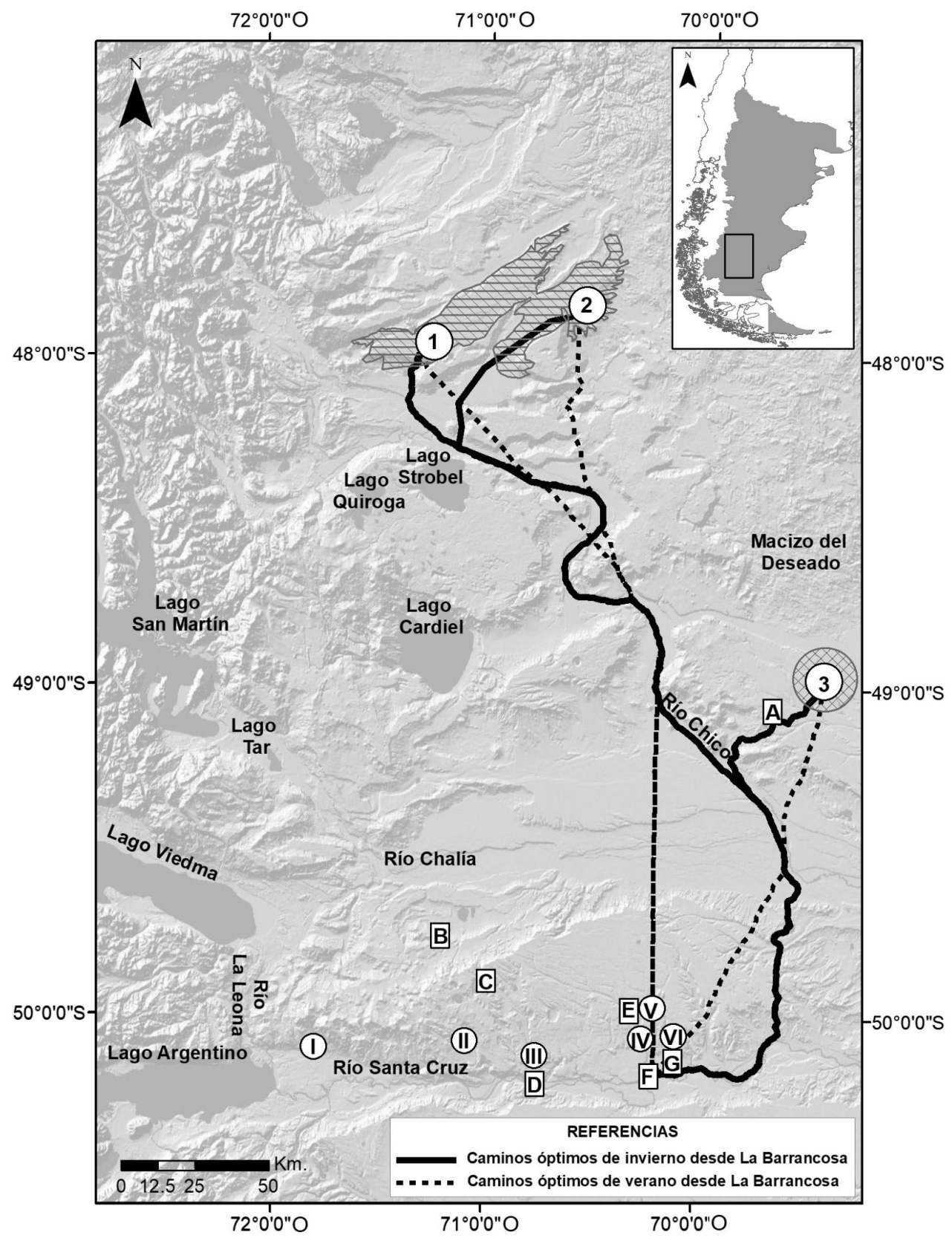

Fig. 5. Caminos óptimos de invierno y verano desde La Barrancosa (curso inferior del cañadón Yaten Guajen). Referencias: 1: PDA, 2: CAPDA, 3: 17 de Marzo; I: La Laurita-El Rincón, II: El Lechuza, III: El Cordero; IV: Yaten Guajen, V: Mercerá, VI: Kolian Kaike; A: Cardiel Chico, B: María Elena, C: La Betty, D: La Fructuosa, E: Yaten Guajen, F: La Barrancosa, G: Güent Aike.

y Strobel, mientras que el que conecta este espacio con el CAPDA pasa a $26 \mathrm{~km}$ al este del lago Cardiel y a $33 \mathrm{~km}$ al este del lago Strobel. Por otra parte, los caminos de invierno desde este sector del espacio a PDA y CAPDA pasan a $39 \mathrm{~km}$ al este del lago Cardiel y a $17 \mathrm{~km}$ al este del lago Strobel. Con respecto al curso medio y superior del cañadón Yaten Guajen, en verano existe una diferencia 


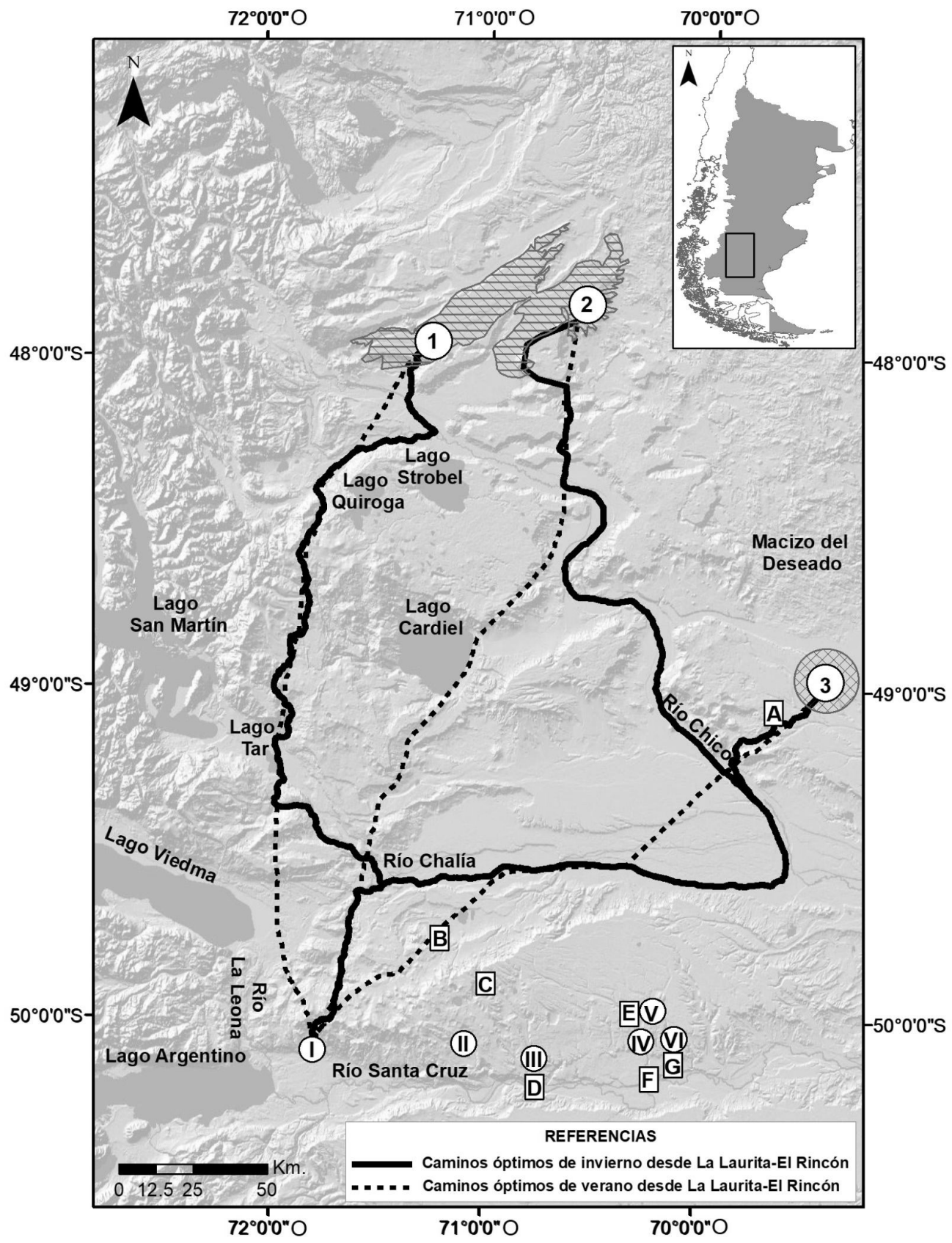

Fig. 6. Caminos óptimos de invierno y verano desde La Laurita-El Rincón. Referencias: 1: PDA, 2: CAPDA, 3: 17 de Marzo; I: La Laurita-El Rincón, II: El Lechuza, III: El Cordero; IV: Yaten Guajen, V: Mercerá, VI: Kolian Kaike; A: Cardiel Chico, B: María Elena, C: La Betty, D: La Fructuosa, E: Yaten Guajen, F: La Barrancosa, G: Güent Aike.

de más de $100 \mathrm{~km}$ entre la distancia a la fuente de 17 de Marzo $(134 \mathrm{~km})$ y las de PDA $(249 \mathrm{~km}$ ) y CAPDA $(252 \mathrm{~km})$. En invierno esta diferencia es aún mayor, oscilando entre 196 km (17 de Marzo), 385 km
(PDA) y 400 km (CAPDA) (Tabla 3). Por otra parte, los caminos óptimos de verano desde este sector a PDA pasan a $28 \mathrm{~km}$ al este del lago Cardiel y a 12 $\mathrm{km}$ al este del lago Strobel, pasando más alejados de 


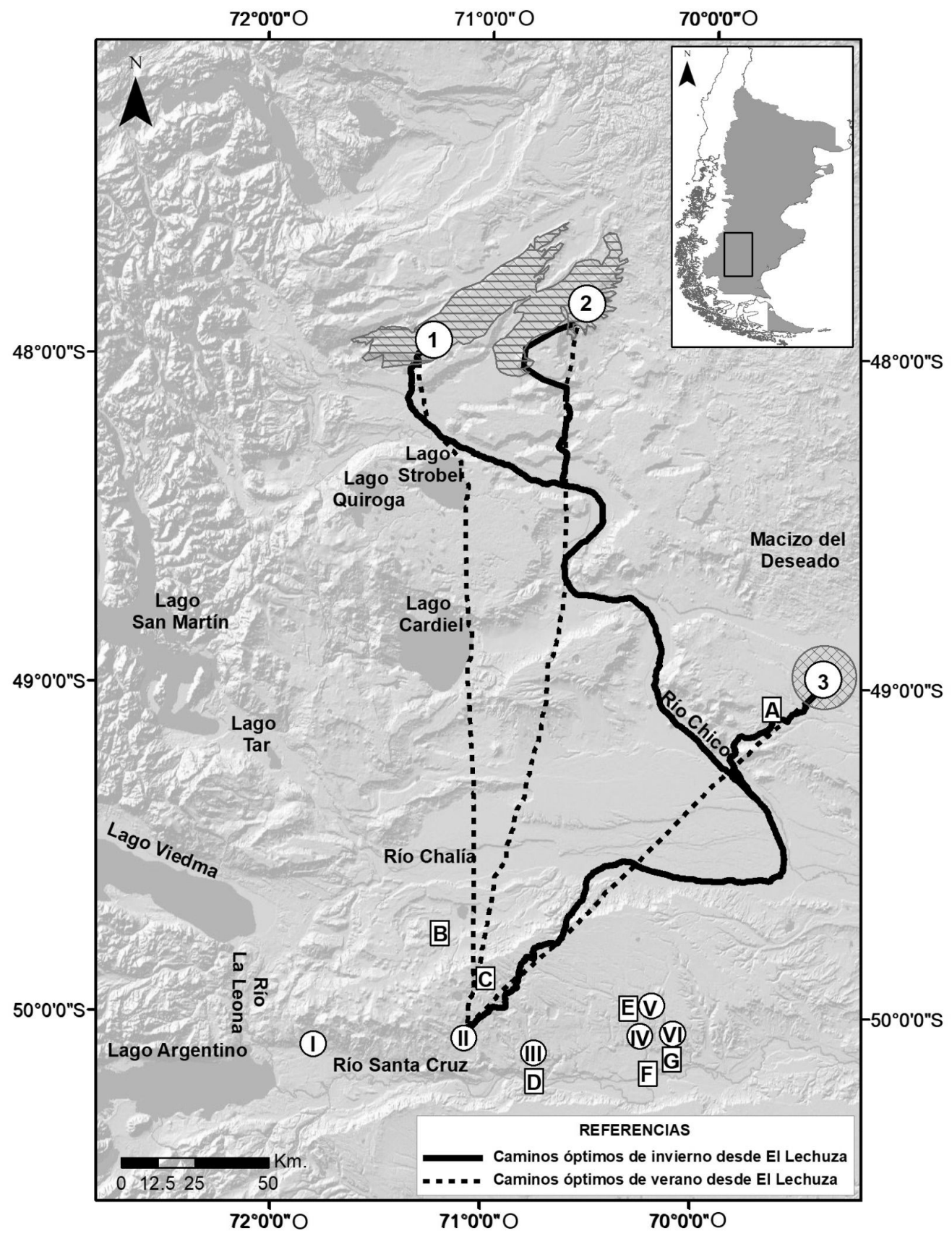

Fig. 7. Caminos óptimos de invierno y verano desde El Lechuza, curso superior y medio.

Referencias: 1: PDA, 2: CAPDA, 3: 17 de Marzo; I: La Laurita-El Rincón, II: El Lechuza, III: El Cordero, IV: Yaten Guajen, V: Mercerá, VI: Kolian Kaike; A: Cardiel Chico, B: María Elena, C: La Betty, D: La Fructuosa, E: Yaten Guajen, F: La Barrancosa, G: Güent Aike.

las cuencas bajas en el caso de los caminos a CAPDA (Fig. 4). Por otro lado, los caminos de invierno desde este sector a PDA y CAPDA pasan a $39 \mathrm{~km}$ al este del lago Cardiel y a $2 \mathrm{~km}$ al este del lago Strobel.
Por último, con respecto a la obsidiana negra recuperada en superficie en los cursos inferiores de los cañadones, existe una diferencia importante en su frecuencia $(1,14 \%$ en el caso 
Tabla 3. Frecuencia de artefactos líticos de obsidiana negra de superficie y estratigrafía en relación con la distancia a PDA, CAPDA y 17 de Marzo. Referencias: Proc.: procedencia; Dist.: distancia; eucl.: euclidiana; km: kilómetros; PDA. Pampa del Asador; CAPDA. Cono aluvial de Pampa del Asador; SD. Sin datos; S.: superficie; E.: estratigrafía; Hol: Holoceno; Tempr.: Temprano; Ref.: Referencias; LL: La Laurita; ER: El

Rincón; EL: cañadón El Lechuza; YG: cañadón Yaten Guajen; csm: curso superior y medio, ci: curso inferior.

\begin{tabular}{|c|c|c|c|c|c|c|c|c|c|c|c|}
\hline Proc. & $\begin{array}{c}\text { Porcentaje } \\
\text { y total de la } \\
\text { muestra }\end{array}$ & $\begin{array}{l}\text { Dist. } \\
\text { eucl. } \\
(\mathrm{km}) \\
\text { a } \\
\text { PDA }\end{array}$ & $\begin{array}{l}\text { Dist. } \\
\text { camino } \\
\text { óptimo } \\
\text { invierno } \\
(\mathrm{km}) \text { a } \\
\text { PDA }\end{array}$ & $\begin{array}{l}\text { Dist. } \\
\text { camino } \\
\text { óptimo } \\
\text { verano } \\
(\mathrm{km}) \text { a } \\
\text { PDA }\end{array}$ & $\begin{array}{c}\text { Dist. } \\
\text { eucl. } \\
(\mathrm{km}) \mathrm{a} \\
\text { CAP- } \\
\text { DA }\end{array}$ & $\begin{array}{c}\text { Dist. } \\
\text { camino } \\
\text { optimo } \\
\text { invierno } \\
(\mathrm{km}) \text { a } \\
\text { CAPDA }\end{array}$ & $\begin{array}{c}\text { Dist. } \\
\text { camino } \\
\text { óptimo } \\
\text { verano } \\
(\mathrm{km}) \mathrm{a} \\
\text { CAPDA }\end{array}$ & $\begin{array}{c}\text { Dist. } \\
\text { eucl. } \\
(\mathrm{km}) \mathrm{a} \\
17 \mathrm{de} \\
\text { Marzo }\end{array}$ & $\begin{array}{c}\text { Dist. } \\
\text { camino } \\
\text { óptimo } \\
\text { invierno } \\
(\mathrm{km}) \text { a } 17 \\
\text { de Marzo }\end{array}$ & $\begin{array}{c}\text { Dist. } \\
\text { camino } \\
\text { óptimo } \\
\text { verano } \\
(\mathrm{km}) \text { a } \\
17 \text { de } \\
\text { Marzo }\end{array}$ & Ref. \\
\hline LL- ER & $\begin{array}{c}\text { S: } 13,17 \% \\
\text { (n 387) } \\
\text { E: SD }\end{array}$ & 230 & 358 & 256 & 258 & 480 & 278 & 208 & 316 & 222 & $\begin{array}{c}\text { Este tra- } \\
\text { bajo }\end{array}$ \\
\hline EL csm & $\begin{array}{c}\text { S: } 12 \%(\mathrm{n} \\
599) \\
\text { E: } \begin{array}{c}3,1 \%(\mathrm{n} \\
35)\end{array}\end{array}$ & 230 & 441 & 240 & 244 & 415 & 256 & 167 & 251 & 169 & $\begin{array}{c}\text { Franco et } \\
\text { al. } 2007 \mathrm{a}, \\
2014\end{array}$ \\
\hline EL ci & $\begin{array}{c}\text { S: } 1,14 \% \\
\text { (n 871) } \\
\text { E.: SD }\end{array}$ & 248 & 487 & 259 & 263 & 502 & 275 & 180 & 298 & 184 & $\begin{array}{c}\text { Vetrisano } \\
2018\end{array}$ \\
\hline YG csm & $\begin{array}{c}\text { S: } 9,6 \% \text { (n } \\
\text { 636) } \\
\text { E: } 23,71 \% \\
\text { (Hol. } \\
\text { Tempr.) } \\
\text { y } 0,76 \% \\
\text { (n } 131 \text { ) al } \\
\text { 12,54\% (n } \\
\text { 576; Hol. } \\
\text { Tardío) }\end{array}$ & 233 & 385 & 249 & 238 & 400 & 252 & 127 & 196 & 134 & $\begin{array}{c}\text { Franco et } \\
\text { al. 2014, } \\
\text { 2015; } \\
\text { Cirigliano, } \\
2016\end{array}$ \\
\hline YG ci & $\begin{array}{c}\text { S: } 6,44 \% \\
\text { (n 978) } \\
\text { E: SD }\end{array}$ & 258 & 414 & 280 & 259 & 429 & 273 & 146 & 225 & 153 & $\begin{array}{l}\text { Este } \\
\text { trabajo }\end{array}$ \\
\hline
\end{tabular}

del cañadón El Lechuza y un $6,44 \%$ en el caso de Yaten Guajen) en relación con lo que ocurre en los cursos superiores y medios (Tabla 3). En el caso del primero de los cañadones mencionados, los modelados de caminos óptimos de verano indican un incremento de valores del orden de 20 $\mathrm{km}$. En cambio, en invierno se encuentran entre los 20 y $40 \mathrm{~km}$. En el caso de Yaten Guajen, los incrementos son menores.

En estratigrafía el patrón es distinto (Tabla 3), pero esto podría estar relacionado con la gran diferencia en los tamaños de las muestras. En el curso medio y superior del cañadón El Lechuza su frecuencia es de alrededor del 3,1\% en el Holoceno tardío (Franco et al. 2014). En su curso inferior, si bien se efectuaron sondeos, aún no se cuenta con datos cuantificados. En el caso del curso superior y medio del cañadón Yaten Guajen, en el sitio Yaten Guajen 1 se registró en estratigrafía, en el Holoceno tardío, un 0,76\% de obsidiana negra (Franco et al. 2014, 2015; Cirigliano, 2016). A una distancia de $200 \mathrm{~m}$ en línea recta se encuentra el sitio Yaten Guajen 12 , en el cual para depósitos correspondientes al Holoceno tardío el 12,54\% de los artefactos es de obsidiana negra (Franco et al. 2015; Cirigliano, 2016). En este sitio, en depósitos correspondientes al Holoceno temprano el porcentaje de obsidiana negra alcanza un 23,71\% (Franco et al. 2015), no encontrándose publicados los datos del total de la muestra. Por último, en el curso inferior de este cañadón no se cuenta con datos cuantificados procedentes de estratigrafía. 
Las frecuencias de obsidiana obtenidas en superficie son consistentes con lo esperado por Pallo y Borrero (2015), quienes utilizaron tanto muestras de superficie como estratigráficas, incluyendo las de este sector del espacio ya publicadas. Cabe señalar, sin embargo, que las diferencias dentro de los distintos sectores podrían estar relacionadas con la funcionalidad o localización de los sitios en ese espacio. Por ejemplo, si bien en general el porcentaje en el caso del curso superior y medio de Yaten Guajen en superficie es del 9,6\%, en el caso de Yaten Guajen 5, sobre un total de 19 artefactos, $3(15,78 \%)$ son de obsidiana negra, mientras que en Yaten Guajen 12, sobre un total de 116 artefactos, $22(18,96 \%)$ están confeccionados en esta materia prima (Cirigliano, 2016). En el caso de Yaten Guajen 12, entonces, el porcentaje es mayor que el esperado por Pallo y Borrero (2015), lo que podría relacionarse con su localización cercana a la confluencia de dos cursos de agua. La baja frecuencia de obsidiana en la desembocadura de estos cañadones podría relacionarse con la mayor distancia involucrada, especialmente en invierno, o con un cambio en la forma de su utilización, siendo necesaria mayor cantidad de información a efectos de evaluarlo.

Las muestras estratigráficas no presentan un patrón de decrecimiento oeste-este, lo que puede relacionarse con el tamaño de las muestras. Los mayores porcentajes corresponden al cañadón Yaten Guajen. En el Holoceno tardío, las frecuencias son altas en el sitio Yaten Guajen 12, encontrándose dentro de lo esperado por Pallo y Borrero, alcanzando el 12,54\% (Franco et al. 2015). En el caso del sitio Mercerat 1, ubicado en el cañadón Mercerá (Fig. 1), que desemboca en el curso superior del cañadón Yaten Guajen, sobre un total de 177 artefactos, 22 son de obsidiana negra, lo que representa un $12,42 \%$ de la muestra (Franco et al. 2015). En el Holoceno temprano, el porcentaje de obsidiana negra en este sitio supera el $23 \%$, no contándose con el n total de la muestra.

\section{DISCUSIÓN}

Como se mencionó, los resultados geoquímicos obtenidos no respaldan la idea de la existencia de otra fuente primaria de obsidiana negra o de una fuente secundaria con una composición geoquímica diferente en el espacio comprendido entre el río Chico y la margen norte de la cuenca del río Santa Cruz.

En los cañadones del norte del río Santa Cruz, las distancias involucradas para la obtención de esta materia prima exceden el rango de acción de poblaciones etnográficas a estas latitudes para el caso de PDA y su cono aluvial (Tabla 3), con la única excepción de las distancias euclidianas y caminos de verano desde Cardiel Chico, que se encuentran dentro de las distancias esperadas para movimientos logísticos en estas latitudes (Kelly, 2011). Por otra parte, todos los caminos de verano a 17 de Marzo se encuentran dentro del rango de distancias que se espera recorran los cazadores-recolectores a estas latitudes. Sin embargo, el registro arqueológico se forma a una escala diferente que el etnográfico y, en este sentido, a través del tiempo la depositación de artefactos habría generado fuentes terciarias de esta materia prima (sensu Church, 1994). La presencia de reclamación -al menos en un casosustentaría esta idea. Su frecuencia, sin embargo, es baja en el registro arqueológico. Por otro lado, como se observa en la tabla 1 , la presencia de corteza en las muestras de obsidiana recuperadas -en un caso casi un guijarro completo- tiende a apoyar el transporte de nódulos de esta materia prima (Civalero \& Franco, 2003).

Las frecuencias de las muestras de superficie de obsidiana negra en los cursos medio y superior de los cañadones muestran un decrecimiento de oeste a este, lo que podría sugerir el mayor acceso a esta materia prima en los espacios más occidentales que en los más orientales. La existencia de semejanzas en el arte rupestre presente en los cañadones del río Santa Cruz y la meseta del Strobel, zona rica en recursos en verano (Belardi \& Goñi, 2006; Goñi et al. 2006; entre otros), localizada próxima a PDA, apunta a relaciones entre estos espacios (Gradin et al.1979; Gradin, 2000, 2001; Belardi \& Goñi, 2006; Franco, 2008; Goñi et al. 2006, 2009; Re, 2010; Franco et al. 2014). Sin embargo, en todos los casos, tanto para los caminos de verano e invierno, como para las distancias euclidianas, las longitudes son menores a 17 de Marzo que a PDA o CAPDA (Tabla 3).

En el caso de La Laurita-El Rincón, la diferencia en distancias entre las fuentes de 17 de Marzo y PDA es de de 34 km en verano (222 versus 
$256 \mathrm{~km}$ ) y de aproximadamente $40 \mathrm{~km}$ en invierno (316 versus $358 \mathrm{~km}$ ). Dado que la diferencia en distancia no es proporcionalmente tan grande, podríamos esperar que se priorice el traslado hacia aquella fuente donde el recurso presentaba nódulos más grandes y en mayor frecuencia, es decir, PDA. Este razonamiento implicaría que la obsidiana es un recurso muy valorado por las poblaciones cazadoras-recolectoras en este espacio. Si bien éste puede ser el caso, otro factor atractor lo podría constituir la presencia de las cuencas bajas y la meseta del Strobel, lo que ha sido defendido por distintos investigadores (Belardi \& Goñi, 2006; Goñi et al. 2006; entre otros). En este sentido, debe recordarse que los caminos desde La LauritaEl Rincón hacia PDA y CAPDA pasan cerca de las cuencas bajas y siguen el área de circulación que coincide con la actual ruta nacional 40 (Fig. 6), lo que sugiere que este espacio en general haya podido resultar atractivo para los antiguos cazadores-recolectores.

En el caso del curso medio y superior de El Lechuza, sólo los caminos de verano a PDA pasan por la margen este de los lagos Cardiel y Strobel (Fig. 7). En este caso, la diferencia en distancia a 17 de Marzo y a PDA es aproximadamente 70 km (169 km a 17 de Marzo y 240 km a PDA) y podrían existir otras fuentes de aprovisionamiento de obsidiana aún no localizadas entre ambas. La semejanza en diseños grabados entre este espacio y la meseta del Strobel sugiere que al menos algunos desplazamientos tuvieron lugar en esa dirección. Esto tendería a sustentar que algunos sectores localizados hacia el oeste puedan haber sido atractivos para las poblaciones humanas, debido a la mayor disponibilidad de humedad, existencia de recursos en relación con lagos, etc. (por ejemplo, Belardi \& Goñi, 2006; Goñi et al. 2006)-, favoreciéndose estas vías de circulación. Los resultados obtenidos no permiten descartar el aprovisionamiento en espacios intermedios entre 17 de Marzo y PDA o CAPDA, donde otras fuentes podrían localizarse y podrían probablemente ser parte de un aprovisionamiento "embedded" (Binford, 1979) durante estos desplazamientos.

Como se ha mencionado, la frecuencia de obsidiana negra en superficie en el curso superior y medio del cañadón Yaten Guajen se encuentra disminuida por la gran cantidad de recolecciones por pobladores locales. En este sentido, llama la atención la mayor abundancia de obsidiana negra en estratigrafía que en superficie en este espacio. $\mathrm{Su}$ frecuencia es alta en el Holoceno temprano (Tabla 3). En el Holoceno tardío, algunas de las frecuencias de obsidiana negra utilizadas en este cañadón son mayores a las registradas hacia el oeste, alcanzando porcentajes mayores al $12 \%$. Cabe señalar que, como puede observarse en la tabla 3 , en este caso hay una gran diferencia en las distancias involucradas en desplazamientos a la fuente distal de 17 de Marzo (134 o 196 $\mathrm{km}$ según se trate de invierno o verano) y las de PDA (entre 249 y $385 \mathrm{~km}$ según sea verano o invierno) o el CAPDA (252 y $400 \mathrm{~km}$ según sea verano o invierno). Contrariamente a lo que ocurre en los casos previos, se trata de diferencias de distancias de más de $100 \mathrm{~km}$. Por otra parte, el camino de invierno a CAPDA pasa a $2 \mathrm{~km}$ al este del lago Strobel, mientras que el de verano pasa a $12 \mathrm{~km}$ al este. En el curso medio y superior del cañadón Yaten Guajen existen motivos grabados semejantes a los localizados en esa meseta, lo que ha permitido sugerir interacción (Gradin, 1976, 2001, 2003; Franco, 2008; Fiore \& Ocampo, 2009; Franco et al. 2014). Sin embargo, las menores distancias a la fuente de 17 de Marzo podrían sugerir: a) que al menos parte de su aprovisionamiento tuvo lugar en la fuente distal de 17 de Marzo o en algún espacio comprendido entre éste y las fuentes de PDA y su cono aluvial, probablemente como estrategia "embedded" (Binford, 1979), o b) que algunas áreas sean favorecidas en la circulación de obsidiana (Molinari \& Espinosa, 1999; Pallo \& Borrero, 2015). El hecho de que, sobre bases macroscópicas, se haya propuesto la circulación de materias primas silíceas con semejanzas morfológicas entre el cañadón Yaten Guajen y el Macizo del Deseado (Franco \& Cirigliano, 2009; Franco et al. 2015) y que la fuente de 17 de Marzo se encuentre muy próxima a él (Franco et al. 2017), tenderían a sustentar la utilización de esta fuente o de otras ubicadas entre ésta y PDA en el marco de la circulación habitual de los cazadores-recolectores. Serán necesarios nuevos trabajos a efectos de evaluar si éste fue efectivamente el caso. 


\section{AGRADECIMIENTOS}

Esta investigación se realizó en el marco de los proyectos UBACyT 20020170100663BA (2018-2021), 20020130100664BA (2014-2017) y PICT 2015-2038. A las Direcciones de Cultura y Turismo de las Municipalidades de Gobernador Gregores, Comandante Luis Piedra Buena y El Calafate. A la Dirección de Patrimonio de la Provincia de Santa Cruz por autorizar los trabajos. A los propietarios de las estancias 17 de Marzo, Cardiel Chico, La Betty, María Elena, El Rincón, La Laurita, Bi Aike, La Fructuosa, Yaten Guajen, Marta, La Barrancosa y Güent Aike. Queremos agradecer muy especialmente al Lic. Lucas Vetrisano (IMHICIHU-CONICET) por su colaboración en los análisis de laboratorio y por permitir la utilización de la información presentada en el CAELA. A la Dra. Natalia Cirigliano (IMHICIHU-CONICET) por autorizar la utilización de información de su tesis de doctorado, realizada en el marco de los proyectos mencionados. A todos los que participaron en los trabajos de campo. A dos revisores anónimos, cuyos comentarios contribuyeron a mejorar el trabajo y al Comité Editor por el tiempo invertido en el proceso de publicación.

\section{BIBLIOGRAFÍA}

Aguerre, A. M. (2003). La Martita: ocupaciones de 8.000 años en la Cueva 4. En A. Aguerre (Ed.), Arqueología y Paleoambiente en la Patagonia Santacruceña Argentina (pp. 29-61). Buenos Aires: Ediciones del Autor.

Ambrústolo, P., Zubimendi, M. A., \& Stern, C. (2012). Explotación de obsidiana negra en la costa norte de Santa Cruz (Patagonia argentina). Cazadoresrecolectores del Cono Sur, 6, 77-86.

Base de Datos Geográfica | Instituto Geográfico Nacional. Recuperado 20 de agosto de 2019, a partir de http:// www.ign.gob.ar/sig

Belardi, J. B., \& Goñi, R. (2006). Representaciones rupestres y convergencia poblacional durante momentos tardíos en Santa Cruz (Patagonia argentina). El caso de la meseta del Strobel. En D. Fiore \& M. Podestá (Eds.), Tramas en la piedra. Producción y usos del arte rupestre (pp. 85-94). World Archaeological Congress, Buenos Aires: Sociedad Argentina de Antropología y Asociación de Amigos del Instituto de Antropología.

Belardi, J. B., Tiberi, P., Stern, C. \& Súnico, A. (2006). Al Este del Cerro Pampa: ampliación del área de disponibilidad de obsidiana de la Pampa del Asador (Provincia de Santa Cruz). Intersecciones en Antropología, 7, 2736.

Belardi J. B., Carballo Marina F., Bourlot T. \& Re, A. (2009). Paisajes arqueológicos, circulación e interacción en diferentes escalas: una perspectiva desde el lago Tar (provincia de Santa Cruz). En M. Salemme, F. Santiago, M. Álvarez, E. Piana, M. Vázquez \& M. Mansur (Eds.), Arqueología de Patagonia: una mirada desde el último confín, Tomo 1 (pp. 219-232). Ushuaia: Utopías.

Binford, L. R. (1979). Organization and formation processes: looking at curated technologies. Journal of Anthropological Research, 35, 255-273.

Binford, L. R. (1980). Willow smoke and dog's tails: huntergatherer settlement systems and archaeological site formation. American Antiquity, 45(1), 4-20.

Brook, G. A., Franco, N. V., Ambrústolo, P., Mancini, M. V., Wang, L., \& Fernández, P. M. (2015). Evidence of the earliest humans in the Southern Deseado Massif (Patagonia, Argentina), Mylodontidae, and changes in water availability. Quaternary International, 363, 107-125.

Cassiodoro, G., Espinosa, S. L., Flores Coni, J., \& Goñi, R. (2015). Disponibilidad de recursos líticos y movilidad durante el Holoceno tardío en el centro-oeste de la provincia de Santa Cruz. Intersecciones en Antropología, 2, 75-86.

Church, T. (1994). Terms in Lithic Resource Studies: Or, is this a lateritic, silcrete or a ferrunginous Wood-grained chert? En T. Church (Ed.), Lithic Resource Studies: A Sourcebook for Archaeologists (pp. 9-15). Lithic Technology, Special Publication \#3. Tulsa, Oklahoma: Department of Anthropology, University of Tulsa.

Cirigliano, N. (2016). Movilidad de grupos indigenas y aprovechamiento de materias primas entre el extremo sur del Macizo del Deseado y la cuenca del río Santa Cruz durante los últimos 2.000 años (provincia de Santa Cruz, Argentina) Tesis Doctoral inédita. Facultad de Filosofía y Letras, Universidad de Buenos Aires, Buenos Aires. Ms.

Civalero, M. T., \& Franco, N. V. (2003). Early Human Occupations in Western Santa Cruz Province, Southernmost South America. Quaternary International, 109-110, 77-86.

Conolly, J., \& Lake, M. (2006). Geographical Information 
Systems in Archaeology. Cambridge: Cambridge University Press.

Cueto, M. D., Frank, A. D., \& Skarbun, F. (2016). The exploitation of obsidian in the Central Plateau of Santa Cruz, Argentina: Results from La María and Cerro Tres Tetas and a regional perspective. Journal of Lithic Studies, 3(2). Proceedings of the $10^{\text {th }}$ International Symposium on Knappable Materials.

Ericson, J. (1984). Towards the analysis of lithic reduction systems. En J. Ericson \& B. Purdy (Eds.), Prehistoric Quarries and Lithic Production (pp. 11-22). Cambridge: Cambridge University Press.

Espinosa, S. L., \& Goñi, R. A. (1999). ¡Viven!: Una fuente de obsidiana en la Provincia de Santa Cruz. En J. Belardi, P. Fernández, R. Goñi, A. Guráieb \& M. de Nigris (Eds.), Soplando en el Viento. Actas de las III Jornadas de Arqueología de la Patagonia (pp. 177188). Neuquén y Buenos Aires: Instituto Nacional de Antropología y Pensamiento Latinoamericano (INAPL) y Universidad Nacional del Comahue.

Fairén Jiménez, S. (2004). ¿Se hace camino al andar? Influencia de las variables medioambientales y culturales en el cálculo de caminos óptimos mediante SIG. Trabajos de Prehistoria, 61(2), 25-40.

Fiore, D., \& Ocampo, M. (2009). Arte rupestre de la región Margen Norte del río Santa Cruz: una perspectiva distribucional. En M. Salemme, F. Santiago, M. Álvarez, E. Piana, M. Vázquez \& M. Mansur (Eds.), Arqueología de Patagonia: una mirada desde el último confín, Tomo 1 (pp. 499-513). Ushuaia: Utopías.

Franco, N. V. (2004). La organización tecnológica y el uso de escalas espaciales amplias. El caso del sur y oeste de Lago Argentino. En A. Acosta, D. Loponte \& M. Ramos (Eds.), Temas de Arqueología, Análisis Lítico (pp. 101-144). Luján: Universidad Nacional de Luján.

Franco, N. V. (2008). La estructura tecnológica regional y la comprensión de la movilidad humana: tendencias para la cuenca del río Santa Cruz. En. L. A. Borrero \& N. V. Franco (Comps.). Arqueología del extremo sur del continente americano. Resultados de nuevos proyectos (pp. 119-154). Buenos Aires: Instituto Multidisciplinario de Historia y Ciencias Humanas (CONICET).

Franco, N. V. (2013). ¿Es posible diferenciar la existencia de grupos humanos con áreas de circulación distintas en el extremo sur de Patagonia durante el Holoceno Tardío? En A.F. Zangrando, R. Barberena, A. Gil, G. Neme, M. Giardina, L. Luna, C. Otaola, S. Paulides, L. Salgán \& A. Tívoli (Eds.), Tendencias teórico-metodológicas y casos de estudio en la Arqueología de la Patagonia (pp. 363-370). San Rafael: Sociedad Argentina de Antropología, Museo de Historia Natural de San Rafael e INAPL.

Franco, N. V. \& Cirigliano, N. A. (2009). Materias primas y movilidad humana entre las cuencas de los ríos Santa Cruz y Chico (provincia de Santa Cruz, Argentina). Primeros resultados. En M. Salemme, F. Santiago, M. Álvarez, E. Piana, M. Vázquez \& M. Mansur (Eds.), Arqueología de Patagonia: una mirada desde el último confín, Tomo 1 (pp. 361-368). Ushuaia: Utopías.

Franco, N. V., Otaola, C., \& Cardillo, M. (2007a). Resultados de los trabajos exploratorios realizados en la margen norte del río Santa Cruz (provincia de Santa Cruz, Argentina). En F. Morello, M. Martinic, A. Prieto \& G. Bahamonde (Eds.), Arqueología de Fuego-Patagonia. Levantando Piedras, desenterrando huesos...y develando arcanos (pp. 541-553). Punta Arenas: Centro de Estudios del Cuaternario, Fuego, Patagonia y Antártica (CEQUA).

Franco, N. V., Cardillo, M., Otaola, C., Arregui, N., \& Gaal, E. (2007b). Tendencias preliminares en el registro arqueológico del curso medio y superior de arroyo El Lechuza, provincia de Santa Cruz, Argentina. Intersecciones en Antropología, 8, 271-285.

Franco, N. V., Martucci, M., Ambrústolo, P., Brook, G. A., Mancini, M. V., \& Cirigliano, N. (2010). Ocupaciones humanas correspondientes a la transición PleistocenoHoloceno al sur del Macizo del Deseado: el área de La Gruta (provincia de Santa Cruz, Argentina). Relaciones de la Sociedad Argentina de Antropología, XXXV, 301-308.

Franco, N. V., Cirigliano, N. A., Fiore, D., Ocampo, M., \& Acevedo, A. (2014). Las ocupaciones del Holoceno tardío en los cañadones basálticos del norte del río Santa Cruz (Patagonia, Argentina). Intersecciones en Antropología, 15, 377-389.

Franco, N. V., Cirigliano, N. A., Vetrisano, L., \& Ambrústolo, P. (2015). Raw material circulation at broad scales in Southern Patagonia (Argentina): the cases of the Chico and Santa Cruz river basins. Quaternary International, 375, 72-83.

Franco, N. V., Brook, G. A., Cirigliano, N. A., Stern, C., \& Vetrisano, L. (2017). 17 de Marzo (Santa Cruz, Argentina): A new distal source of Pampa del Asador type black obsidian and its implications for understanding hunter-gatherer behavior in Patagonia. Journal of Archaeological Science Reports, 12, 232243. 
Franco, N. V., Borrero, L. A., \& Lucero, G. (2019). Human dispersal in the Atlantic slope of Patagonia and the role of lithic availability. PaleoAmérica, 5(1), 88-104.

Gilio, B. (2017). Circulación de cazadores-recolectores en la margen norte del río Santa Cruz y espacios basálticos septentrionales (Patagonia, Argentina) durante el Holoceno Tardio: una aproximación a partir de los Sistemas de Información Geográfica (SIG). Tesis de Licenciatura inédita, Universidad de Buenos Aires, Argentina. Facultad de Filosofía y Letras, Universidad de Buenos Aires, Buenos Aires. Ms.

Gilio, B., Skarbun, F., \& Franco, N. V. (2016). Modelado de vías de movilidad entre sitios de cazadores-recolectores en la margen norte del río Santa Cruz (Patagonia, Argentina) durante el Holoceno Tardío. GeoSIG, 8 (8), 51-73.

Glascock, M. D., \& Ferguson, J. R. (2012). Report on the Analysis of Obsidian Source Samples by Multiple Analytical Methods. Archaeometry Laboratory. Columbia: University of Missouri.

Goñi, R. A., Belardi, J. B., Espinosa, S. L., Savanti, F., Molinari, R., Barrientos, G., Bourlot, T. J.,... \& García Guráieb, S. (2006). Cazadores recolectores de las cuencas de los lagos Cardiel y Strobel: su integración a la dinámica poblacional tardía del sur de Patagonia. Cazadores Recolectores del Cono Sur, 1, 57-66.

Goñi, R., Belardi, J. B., Espinosa, S. L., Cassiodoro, G., Bourlot, T., García Guráieb, S., Re, A.,... de Santa Cruz. En S. Mirelman, A. Tauber, M. E. Palacios, P. Campan, P. Álvarez \& E. Luque (Eds.), Estado actual de las investigaciones realizadas sobre Patrimonio Cultural en Santa Cruz (pp. 195-202). Río Gallegos: Dirección de Patrimonio Cultural. Subsecretaría de Cultura de Santa Cruz.

Gradin, C. (1976). Parapetos de piedra y grabados rupestres de la meseta del lago Buenos Aires. Actas y Memorias del IV Congreso Nacional de Arqueología Argentina (Primera parte). Revista del Museo de Historia Natural de San Rafael, II(1/4), 315-337.

Gradin, C. (1983). Los grabados rupestres del sur de la Patagonia. Revista Patagónica, 2(8), 13-18.

Gradin, C. (2000). Más allá y más acá del Río Santa Cruz. Buenos Aires: Nuevo Offset.

Gradin, C. (2001). El arte rupestre de los cazadores de guanaco de la Patagonia. En E. Berberián \& A. Nielsen (Eds.), Historia Argentina Prehispánica, Tomo 2, (pp. 839874). Córdoba: Brujas.

Gradin, C. (2003). Grabados de la Estancia "La Flecha", Gobernador Gregores-Provincia de Santa Cruz. En A. M. Aguerre (Comp.), Arqueología y Paleoambiente en la Patagonia Santacruceña Argentina, (pp. 121-137). Buenos Aires: Nuevo Offset.

Gradin, C. J., Aschero, C. A., \& Aguerre, A. M. (1979). Arqueología del área Río Pinturas (Provincia de Santa Cruz). Relaciones de la Sociedad Argentina de Antropología, XIII, 183-227.

Ingbar, E. (1994). Lithic material selection and technological organization. En P. Carr (Ed.), The Organization of North American Chipped Stone Tool Technologies (pp. 45-56). Ann Arbor: International Monographies in Prehistory.

Kelly, R. (2011). Obsidian in the Carson Desert: Mobility or Trade? In R. Hughes (Ed.), Investigating Prehistoric Trade and Exchange in Western North America, (pp. 189-200). Salt Lake City: University of Utah Press.

Kelly, R. (2013). The Lifeways of Hunter-Gatherers. The Foraging Spectrum. Cambridge: Cambridge University Press.

López Romero, R. (2005). Cálculo de rutas óptimas mediante SIG en el territorio de la ciudad Celtibérica de Segeda. Propuesta metodológica. Saldvie, 5, 95-111.

Magnin, L. (2013). Localidades arqueológicas del norte de la provincia de Santa Cruz y vías de movilidad entre ellas. Los SIG en la integración de paisajes arqueológicos regionales. En A. F. Zangrando, R. Barberena, A. Gil, G. Neme, M. Giardina, L. Luna, C. Otaola, S. Paulides, L. Salgán \& A. Tívoli (Eds.), Tendencias teóricometodológicas y casos de estudio en la arqueología de la Patagonia (pp. 9-30). San Rafael: Sociedad Argentina de Antropología, Museo de Historia Natural de San Rafael e INAPL

Meltzer, D. (1989). Was stone exchange among eastern North American paleoindians? En C. Ellis \& J. Lothrop (Eds.), Eastern paleoindian lithic resource use (pp. 11-39). Boulder: Westview Press.

Molinari, R., \& Espinosa, S. L. (1999). Brilla tú, diamante "loco". En J. Belardi, P. Fernández, R. Goñi, A. Guráieb \& M. de Nigris (Eds.), Soplando en el Viento. Actas de las III Jornadas de Arqueología de la Patagonia (pp.189198). Neuquén y Buenos Aires: Instituto Nacional de Antropología y Pensamiento Latinoamericano (INAPL) y Universidad Nacional del Comahue.

Nami, H., Giesso, M., Castro, A., \& Glascock, M. (2018). New analysis of Late Holocene obsidians from Southern Patagonia (Santa Cruz Province, Argentina). Bulletin of the International Association for Obsidian Studies, 57, 13-23.

Pallo, M. C. (2016). Corredores naturales, fuente de obsidiana y estacionalidad: El caso de la circulación humana entre 
Pali Aike y Sierra Baguales (Patagonia Meridional). Chungará Arica, 48(1), 25-37.

Pallo, M. C., \& Borrero, L. A. (2015). ¿Intercambio o Movilidad?: Una Evaluación Sobre el Uso de Escalas de Análisis Espaciales y Curvas de Declinación en Patagonia Centro-Meridional (Argentina). Latin American Antiquity, 26(3), 287-303.

Re, A. (2010). Representaciones rupestres en mesetas altas de la provincia de Santa Cruz. Circulación de información en espacios de uso estacional. Tesis Doctoral inédita. Facultad de Filosofía y Letras, Universidad de Buenos Aires, Buenos Aires. Ms.

Renfrew, C. (1977). Alternative Models for Exchange and Spatial Distribution. En T. Earle \& J. Ericson (Eds.), Exchange systems in Prehistory (pp. 71-89). New York: Academic Press.

Stern, C. R. (1999). Black obsidian from Central-South Patagonia: Chemical Characteristics, Sources and Regional Distribution of Artifacts. En J. Belardi, P. Fernández, R. Goñi, A. Guráieb \& M. de Nigris (Eds.), Soplando en el viento. Actas de las III Jornadas de Arqueología de Patagonia (pp. 221234). Neuquén y Buenos Aires: Instituto Nacional de Antropología y Pensamiento Latinoamericano y Facultad de Humanidades, Universidad Nacional del Comahue.

Stern, C. R. (2000). Sources of obsidian artifacts from the Pali Aike, Fell’s Cave and cañadón La Leona archaeological sites in southernmost Patagonia. En J. B. Belardi, F. Carballo Marina \& S. L. Espinosa (Eds.), Desde el país de los gigantes. Perspectivas arqueológicas en Patagonia (pp. 43-55). Río Gallegos: Universidad Nacional de la Patagonia Austral.

Stern, C. R. (2004). Obsidian in Southern Patagonia: review of the current information. En T. Civalero, P. Fernández \& G. Guráieb (Eds.), Contra viento y marea. Arqueología de Patagonia (pp. 167-176). Buenos Aires: Instituto Nacional de Antropología y Pensamiento Latinoamericano.

Stern, C. R., Mena, F., Aschero, C. A., \& Goñi, R. (1995a). Obsidiana negra de los sitios arqueológicos en la precordillera andina de Patagonia central. Anales del Instituto de la Patagonia, Serie Ciencias Humanas, 23, 111-118.

Stern, C. R., Prieto, A., \& Franco, N. (1995b). Obsidiana negra en sitios arqueológicos de cazadores-recolectores terrestres en Patagonia austral. Anales del Instituto de la Patagonia, Serie Ciencias Humanas, 23, 105-109.

Vetrisano, L. (2018). Variabilidad de métodos de reducción en conjuntos líticos superficiales de la costa norte del río Santa Cruz, localidad arqueológica de Bi Aike, Patagonia Argentina. En R. Cattáneo, A. Izeta, T. Costa \& G. Sario (Comps.), Libro de resúmenes. Primer Congreso Argentino de Estudios Líticos en Arqueología (pp. 57-58). Córdoba: Universidad Nacional de Córdoba. 